\title{
水稻线粒体 $\mathrm{TRNA}^{\mathrm{Trp}}$ 的种属特异性氨酰化
}

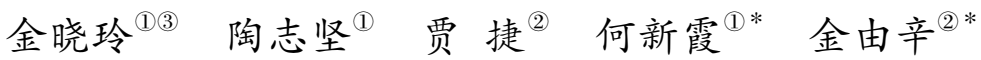

(1) 浙江师范大学化学与生命科学学院, 金华 321004; (2) 中国科学院上海生命科学研究院生物化学与细胞生物学研究所, 分子生物 学国家重点实验室, 上海 200033; (3) 中南林学院环艺学院, 长沙 410004.* 联系人, E-mail: yxjin@ @sibs. ac.cn, jhhxx@zjnu.cn)

摘要 为了研究原核生物和真核生物(细胞质)色氨酰 tRNA 合成酶(TrpRS)对线粒体 $\mathrm{tRNA}{ }^{\mathrm{Tr}}$ 识别的种属 特异性, 构建了 7 个水稻线粒体 $\mathrm{tRNA}^{\mathrm{Trp}}$ 三位点 $(\mathrm{G} 73, \mathrm{U}, \mathrm{U} 2, \mathrm{~A} 68)$ 的单点或多点突变的突变体. 这些突变 基因, 经体外转录后分别用枯草杆菌(B. subtilis)和人色氨酰 tRNA 合成酶(TrpRS)进行氨酰化反应, 并测 定它们的动力学常数. 结果表明, 与野生型水稻线粒体 $\mathrm{tRNA}^{\operatorname{Tr}}$ 相比, 7 个突变体的转录产物被B. subtilis TrpRS 氨酰化的活力分别降低了 53.33\% 99.79\%, 被人 TrpRS 氨酰化的活力却分别提高了 4 330 倍, 其 中以 MPH7(水稻线粒体 $\mathrm{tRNA}^{\mathrm{Tr}}$ 的三碱基 (G73, U72 和 $\mathrm{C} 68$ )全部突变为人 $\mathrm{tRNA}^{\mathrm{Tr}}$ 的三碱基序列)的氨 酰化活力改变最大. 实验结果证明, 与真核生物和原核生物细胞质 $\mathrm{tRNA}^{\mathrm{Tr}}$ 的种族特异性类似, 水稻线 粒体 $\mathrm{tRNA}^{\mathrm{Trp}}$ 的种属特异性元件也主要处于氨基酸接受茎的识别位碱基、第一和第五对碱基对, 亦即识 别位碱基 G73, 氨基酸接受茎上的两个碱基对 G1/U72 和 U5/A68. 本研究为线粒体 tRNATrp 起源于真 细菌的推论提供了实验依据.
\end{abstract}

\section{关键词 种属特异性氨酰化 色氨酸 tRNA 色氨酰 tRNA 合成酶 水稻 线粒体}

蛋白质合成的精确性对于所有生物体的生存都 是必须的. 氨酰 tRNA 合成酶催化相应 tRNA 分子的 氨酰化反应是蛋白质合成忠实性的分子基础 ${ }^{[1]}$. 近年 来, 在 20 种氨酰化系统中, 鉴定出大量 tRNA 分子的 识别元件、特异的功能基团和细微的结构特征, 这对 揭示氨酰 tRNA 合成酶与 tRNA 分子识别的机理起了 重要作用 ${ }^{[2,3]}$. 研究表明, 识别位碱基(N73)在对应于 多种氨基酸的 RNA 操作密码中有关键作用 ${ }^{[4,5]}$, 但它 在各个种属之间并不是严格保守的. 例如, 在甘氨酸 系统中, 真核和古核来源的 $\mathrm{tRNA} \mathrm{Gly}^{\mathrm{Gl}}$ 识别位碱基是 $\mathrm{A} 73$, 而原核来源的 $\mathrm{tRNA}{ }^{\mathrm{Gly}}$ 识别位碱基是 $\mathrm{U} 73^{[6]}$. 同 时, 大肠杆菌甘氨酰 tRNA 合成酶和人甘氨酰 tRNA 合成酶不能交叉识别它们对应的 tRNA ${ }^{\mathrm{Gly}}$ [7]. 这些事 实都表明, 真核和古核的 RNA 操作密码与原核的不 同, 而这种不同是不同种属来源的 tRNA 分子不能交 叉识别的主要原因. 深人研究各种氨酰 tRNA 合成酶 的种族特异性，不仅有其重要的理论意义，也将有助 于新药靶的发现.

已有研究表明, 枯草杆菌色氨酰 tRNA 合成酶识 别的个性元件是识别位碱基 G73 和反密码子(主要个 性元件, 即影响较大的元件); A1/U72, G5/C68 和 A9 (次要个性元件, 即影响较小的元件). 人(细胞质) $\mathrm{tRNA}^{\mathrm{Trp}}$ 的个性元件是识别位碱基 A73 和反密码子 (主要个性元件); G1/C72, U5/G68(次要个性元件) ${ }^{[5]}$.
由此可见，除反密码子外，其他的个性元件主要位于 $\mathrm{tRNA}^{\mathrm{Trp}}$ 的氨基酸接受茎上. 此外, 真细菌和真核 $\mathrm{tRNA}^{\mathrm{Trp}}$ 的氨酰化反应也存在种属特异性, 它们是 $\mathrm{G} 73 \mathrm{~A}, \mathrm{~A} 1 \mathrm{G} / \mathrm{U} 72 \mathrm{C}$ 和 $\mathrm{G} 5 \mathrm{U} / \mathrm{C} 68 \mathrm{G}^{[8]}$. Xu 等人 ${ }^{[9]}$ 的另一 研究发现, B. subtilis tRNA ${ }^{\mathrm{Trp}}$ 接受茎的 3 个碱基对 (G2/C71, G3/C70 和 G4/C69)被证明为新的种属特异 性元件, 但在真核(细胞质)的 $\mathrm{tRNA}^{\mathrm{Trp}}$ 中, 这 3 个碱基 对在其氨酰化识别中并不重要.

生物体内主要存在 3 种类型的 tRNA, 即原核生 物 tRNA、真核生物 (细胞质)tRNA 和细胞器 tRNA. 目 前, 对于原核生物 $\mathrm{tRNA}^{\mathrm{Trp}}$, 真核生物 (细胞质) $\mathrm{tRNA}^{\mathrm{Trp}}$ 的种属特异性元件的研究已经比较清楚, 但 对于细胞器 $\mathrm{tRNA}^{\mathrm{Trp}}$ 的种属特异性元件的研究很少. 线粒体 tRNA 是一类细胞器 tRNA, 它的结构既不同 于真核生物 (细胞质)的 $\mathrm{tRNA}^{\mathrm{Trp}}$, 也不同于原核生物 的 $\mathrm{tRNA}^{\mathrm{Trp}}$. 检索已知细胞器 $\mathrm{tRNA}^{\mathrm{Trp}}$ 的序列发现, 大 部分已知细胞器 $\mathrm{tRNA}^{\mathrm{Trp}}$ 序列来自植物的线粒体与叶 绿体, 并且叶绿体和线粒体 $\mathrm{tRNA}{ }^{\mathrm{Tr}}$ 的氨基酸接受茎 序列有明显的保守性. 为了了解线粒体 $\mathrm{tRNA}^{\mathrm{Trp}}$ 在与 色氨酰 tRNA 合成酶识别中的种属特异性, 本研究设 计了水稻线粒体 $\mathrm{tRNA}^{\mathrm{Trp}}$ 单点和多点突变的 7 个突变 体, 并用枯草杆菌和人的色氨酰 tRNA 合成酶(TrpRS) 来测定它们的氨酰化活力, 以了解线粒体 $\mathrm{tRNA}^{\mathrm{Trp}}$ 与不同种族 $\operatorname{TrpRS}$ 的识别特性, 研究线粒体 $\mathrm{tRNA}^{\mathrm{Trp}}$ 
的种属特异性元件, 并研究这些位点在种属特异性 进化过程中的功能.

\section{1 材料和方法}

(i ) 质粒和试剂. 用于高表达枯草杆菌色氨酰 tRNA 合成酶的质粒 $\mathrm{pKSW} 1^{[5]}$ 由香港科技大学薛红博 士惠赠. 人色氨酰 tRNA 合成酶的高表达质粒 pET24a(+)-HTrpRS 由上海生命科学研究院分子生物 学重点实验室构建 ${ }^{[10]}$. T4 DNA 连接酶、限制性内切 酶 $B s t \mathrm{O}$ I 和 RiboMAX Large Scale RNA Production System-T7 购自 Promega (Madison, WI). L- $\left[5-{ }^{3} \mathrm{H}\right]$ Tryptophan 购自 Amersham Pharmacia 公司(Piscataway, NJ). L-Tryptophan购自 Gibco BRL 公司(Gaithersburg, MD). Ni-NTA-agarose 购自 Qiagen 公司(Chatsworth, CA).

(ii) 引物和模版. $5^{\prime}$ 端引物为通用引物, 用于 在 tRNA 基因前加上 $\mathrm{T} 7$ 启动子, 使得转录从 +1 位 (表 1 中用斜体标出), 亦即从 $\mathrm{tRNA}{ }^{\operatorname{Tr}}$ 基因第一位起始. $3^{\prime}$ 引物为特异引物, 以导人不同的突变 (表 1 中突变位 点的 6 核苗酸序列均用黑斜体标出). 该序列中与模 板 3'端序列反向互补的部分用下横线标出. 所有 3'引 物中均含限制性内切核酸酶 $B s t \mathrm{OI}$ (该酶剪切后的模 板, 刚好使转录终止在 CCA 末端处, 获得成熟的 tRNA 末端)和 Hind III 酶切位点. 模板为 tDNA ${ }^{\mathrm{Trp}}$, 即 tRNA $\mathrm{R}^{\mathrm{Trp}}$ 结构基因的正义链(序列来自 tRNA 序列库, ID为 DW4460, http://www.staff.uni-bayreuth.de/ btc914/search/ index.html)中的 10 66 位. 该序列中与 5'引物同源部分 以及与 $3^{\prime}$ 引物反向互补部分同样用下横线标出. 引物 和模板的序列见表 1. 所有模板和引物 DNA 均由本 课题组合成. 含野生型人 $\mathrm{tRNA}^{\mathrm{Trp}}$ 基因的质粒由本室 提供. （iii）含线粒体 $\mathrm{tRNA}^{\mathrm{Trp}}$ 突变基因的质粒构建. 各种线粒体 $\mathrm{tRNA}^{\mathrm{Tr}}$ 突变基因的质粒构建程序参照 Sampson 和 Uhlenbeck 的报道 ${ }^{[11]}$. 以表 1 中的模板序 列作为 PCR 模板, 用 $5^{\prime}$ 引物和引物 1 进行 PCR, 所得 产物即为 MPH1 突变 $\mathrm{tRNA}{ }^{\mathrm{Trp}}$ 基因. 其他突变体以相 同的 PCR 方法用不同的引物构建. $50 \mu \mathrm{L}$ 反应体积含 两种引物各 $0.4 \mu \mathrm{mol} / \mathrm{L}$, 模板 $1 \mathrm{nmol} / \mathrm{L}, \mathrm{MgCl}_{2} 2$ $\mathrm{mmol} / \mathrm{L}$, Taq DNA 聚合酶 $1 \mathrm{U}(\mathrm{TaKaRa})$. PCR 反应条 件为: $94^{\circ} \mathrm{C}, 1 \mathrm{~min}, 60^{\circ} \mathrm{C}, 45 \mathrm{~s}, 72^{\circ} \mathrm{C}, 30 \mathrm{~s} ; 33$ 个循环; $72^{\circ} \mathrm{C}$ 延伸 $10 \mathrm{~min}$. 扩增片段长度为 $109 \mathrm{bp}$. PCR 产物 经低熔点琼脂糖凝胶纯化回收, 克隆到 pGEM-T 载 体(Promega 公司)(有 $S f i$ I 酶切位点)中. DNA 序列经 测序验证(Eppendorf 公司, MasterCycler Gradient). 用 质粒抽提试剂盒小量制备鉴定正确的质粒, 经 Sfi I 和 Hind III 双酶切后亚克隆到 pGEM-9Zf (-)载体中. 图 1 是线粒体和人的 2 种野生型 $\mathrm{tRNA}^{\mathrm{Trp}}$ (图 1(a)和(i)) 和 7 种突变体(图 1(b) (h))的二级结构示意图.

(iv) $\mathrm{tRNA}^{\mathrm{Tr}}$ 的体外转录. 所有的 $\mathrm{tRNA}^{\mathrm{Tr}}$ 突变 体均用其突变基因由体外转录方法制备 ${ }^{[10]} .50 \mu \mathrm{L}$ 转 录体系中含 $10 \mu \mathrm{g}$ 经 $B s t \mathrm{O}$ I 酶切线性化的 $\mathrm{tRNA}^{\mathrm{Trp}}$ 基 因转录模板, $40 \mathrm{mmol} / \mathrm{L}$ Tris- $\mathrm{HCl}(\mathrm{pH} 8.0), 15 \mathrm{mmol} / \mathrm{L}$ $\mathrm{MgCI}_{2}, 5 \mathrm{mmol} / \mathrm{L} \mathrm{DTT}, 500 \mathrm{mmol} / \mathrm{LBSA}$ (无核酸酶), $1.5 \mathrm{mmol} / \mathrm{L}$ NTPs, $40 \mathrm{U}$ RNasin (Promega)和 $200 \mathrm{U}$ T7 RNA 聚合酶(Ampharmacia). 反应体系在 $37^{\circ} \mathrm{C}$ 保温 2 $\mathrm{h}$ 后加人 $10 \mathrm{U}$ DNase, $37^{\circ} \mathrm{C}$ 再保温 $30 \mathrm{~min}$ 以水解 DNA 模板. 转录产物经 $10 \%$ 聚丙烯酰胺变性胶电泳纯化. 在进行氨酰化反应之前, 转录产物于 $70^{\circ} \mathrm{C}$ 加热 $2 \mathrm{~min}$, 缓慢退火至室温, 使 $\mathrm{tRNA}^{\mathrm{Tr}}$ 形成正确的高级结构.

( V ) 酶的纯化和活力测定. 枯草杆菌色氨酰 tRNA 合成酶和人色氨酰 tRNA 合成酶的纯化参照文

表 1 用于扩增的水稻线粒体 $\mathrm{tRNA} \mathrm{Ar}^{\mathrm{Trp}}$ 和突变体的引物和模板

\begin{tabular}{|c|c|c|}
\hline & 序列 $\left(5^{\prime} \rightarrow 3^{\prime}\right)^{a)}$ & 作用 \\
\hline 5'引物 & CTCTAATACGACTCACTATAGCGCTCTTAGTTCAGTTCGGTAGAACGTG & PCR 5'通用引物 \\
\hline 标准 3’引物 & TCCCAAGCTTCCTGG $C A C G C T C T G T A G G A T T T G A A C C T A C G A C A$ & 野生稻线粒体 $\mathrm{tRNA}{ }^{\mathrm{Tr}}$ 扩增引物 \\
\hline 引物 1 ( $3^{\prime}$ 引物) & TCCCAAGCTTCCTGG TACGCTCTGTAGGATTTGAACCTACGACA & MPH1 扩增引物 \\
\hline 引物 2 (3'引物) & TCCCAAGCTTCCTGGCGCGCTCTGTAGGATTTGAACCTACGACA & $\mathrm{MPH} 2$ 扩增引物 \\
\hline 引物 3 (3'引物) & TCCCAAGCTTCCTGG $C A C G C G C T G T A G G A T T T G A A C C T A C G A C A$ & MPH3 扩增引物 \\
\hline 引物 4 (3'引物) & TCCCAAGCTTCCTGG TGCGCTCTGTAGGATTTGAACCTACGACA & MPH4 扩增引物 \\
\hline 引物 5 (3'引物) & TCCCAAGCTTCCTGG TACGCCCTGTAGGATTTGAACCTACGACA & MPH5 扩增引物 \\
\hline 引物 6 (3’引物) & TCCCAAGCTTCCTGGCGCGCCCTGTAGGATTTGAACCTACGACA & MPH6 扩增引物 \\
\hline 引物 7 (3’引物) & TCCCAAGCTTCCTGG TGCGCCCTGTAGGATTTGAACCTACGACA & MPH7 扩增引物 \\
\hline 模板 & $\begin{array}{l}\text { GTTCAGTTCGGTAGAACGTGGGTCTCCAAAACCCAATGTCGTAGGTT- } \\
\text { CAAATCCTAC }\end{array}$ & PCR \\
\hline
\end{tabular}

a) 黑斜体表示突变位点的 6 核苷酸序列 

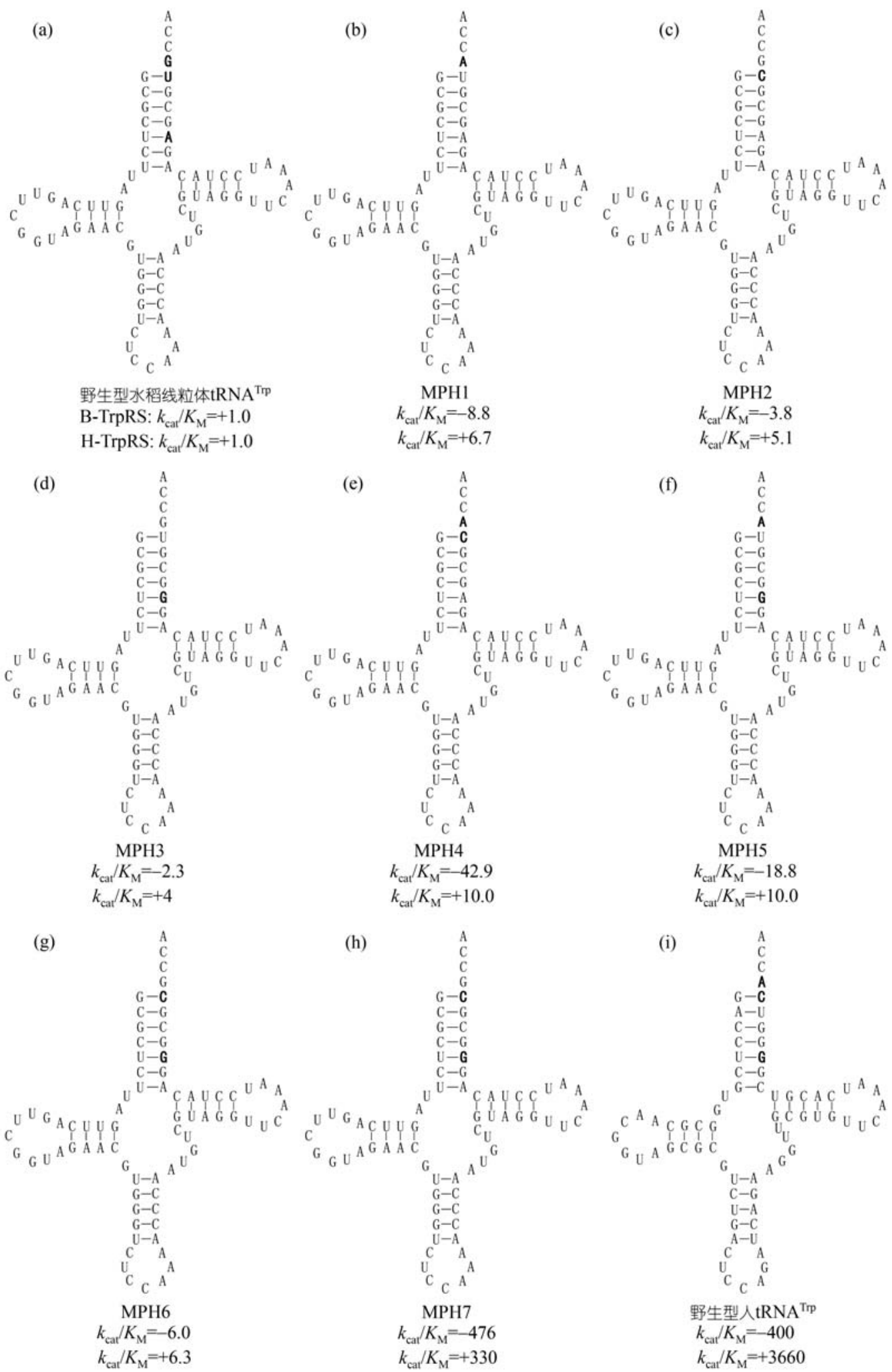

图 1 野生型水稻线粒体 $\mathrm{tRNA}^{\mathrm{Trp}}$, 人 $\mathrm{tRNA}^{\mathrm{Trp}}$ 和 7 个突变体的二级结构及氨酰化反应动力学参数 突变体的突变位点和它们野生型 $\mathrm{TRNA}^{\mathrm{Tr}}$ 的相应位点用粗黑体表示. 假设野生型水稻线粒体 $\mathrm{tRNA}{ }^{\operatorname{Trp}}$ 被其 $\operatorname{TrpRS}$ 催化的 $k_{\mathrm{cat}} / K_{\mathrm{M}}$ 为 1.0. 分析用的 $\mathrm{tRNA}^{\mathrm{Trp}}$ 浓度从 0.1 到 $20 \mu \mathrm{mol} / \mathrm{L}$, 酶的浓度为 3 或 $4 \mathrm{nmol} / \mathrm{L} / \mathrm{B}-\mathrm{TrpRS}$ 和 H-TrpRS 分别为 B. subtilis 和人的 TrpRS 
献[10,12]. 枯草杆菌色氨酰 tRNA 合成酶的氨酰化活 力测定在 $22^{\circ} \mathrm{C}$ 进行, $50 \mu \mathrm{L}$ 反应混和物中含有 4 $\mathrm{mmol} / \mathrm{L}$ ATP, $1 \mathrm{mmol} / \mathrm{L}$ DTT, $1 \mu \mathrm{Ci} \mathrm{L}-\left[5-{ }^{3} \mathrm{H}\right]$ 色氨酸, $40 \mathrm{mmol} / \mathrm{L}$ 醋酸镁, $140 \mathrm{mmol} / \mathrm{L}$ Tris- $\mathrm{HCl}(\mathrm{pH} 7.8), 0.02$ $\mu \mathrm{mol} / \mathrm{L}$ 体外转录的人 $\mathrm{tRNA}^{\mathrm{Trp}[13]}$. 人色氨酰 tRNA 合 成酶的氨酰化活力测定在 $30^{\circ} \mathrm{C}$ 进行, $50 \mu \mathrm{L}$ 反应混和 物中含 $4 \mathrm{mmol} / \mathrm{L}$ ATP, $0.8 \mathrm{mmol} / \mathrm{L}$ DTT, $1 \mu \mathrm{Ci} \mathrm{L}-\left[5-{ }^{3} \mathrm{H}\right]$ 色氨酸, $8 \mathrm{mmol} / \mathrm{L} \mathrm{MgCI}_{2}, 80 \mathrm{mmol} / \mathrm{L}$ Tris- $\mathrm{HCl}(\mathrm{pH}$ $7.5), 0.02 \mu \mathrm{mol} / \mathrm{L}$ 体外转录的人 tRNA ${ }^{\mathrm{Trp}}$. 滤纸片先用 $5 \mathrm{~g} / \mathrm{L}$ 色氨酸溶液浸泡过夜以竞争性地降低本底, 临 用前烘干. 取 $10 \mu \mathrm{L} \mathrm{tRNA}{ }^{\mathrm{Trp}}$ 色氨酰化反应液点样, 用 $100 \mathrm{~mL}$ 冰预冷的 $50 \mathrm{~g} / \mathrm{L} \mathrm{TCA}$ (含 $5 \mathrm{~g} / \mathrm{L}$ 色氨酸)洗涤 3 次, 再用 $10 \mathrm{~mL} 95 \%$ 乙醇及无水乙醚各洗涤一次, 滤纸用红外干燥后在 Wallac 1490 液闪计数器 (Pharmacia)上对 $\left.{ }^{3} \mathrm{H}\right]$-tryptophan 计数. 一个氨酰化活力 单位定义为在标准反应条件下, 每分钟氨酰化 $1 \mathrm{pmol}$ $\mathrm{tRNA}^{\mathrm{Trp}}$ 所需的酶量. 动力学常数通过 LineweaverBurk 作图得到.

\section{2 结果}

\section{1 含 $\mathrm{tRNA}^{\mathrm{Trp}}$ 突变基因质粒的构建}

所构建 7 种含 $\mathrm{tRNA}^{\mathrm{Trp}}$ 突变基因质粒的大小约 $3.0 \mathrm{~kb}$, 含有一个 $\mathrm{T} 7$ 启动子, 控制 $\mathrm{tRNA}^{\mathrm{Trp}}$ 基因的转 录/这些质粒可以通过体内表达和体外转录, 以用于 进一步研究. 它们分别是识别位碱基、构成氨基酸接 受茎第一个碱基对的 72 位、构成氨基酸接受茎第 5 个碱基对的 68 位的 3 种单碱基突变体(MPH1, MPH2 和 MPH3). 由此 3 种单突变的不同组合而形成的 3 种 双突变体(MPH4, MPH5, MPH6)和此 3 位点同时突变 的突变体 (MPH7), 这 3 个位点在原核生物与真核生物 细胞质 $\mathrm{tRNA}^{\mathrm{Trp}}$ 中, 均与种族特异性有关(图 1). 研究 此 7 种突变体的种族特异性, 可了解线粒体 $\mathrm{tRNA}^{\mathrm{Tr}}$ 与细胞质 $\mathrm{tRNA}^{\mathrm{Tr}}$ 在种族特异性组成上的差异.

\section{2 野生型水稻线粒体和人 $\mathrm{tRNA}^{\mathrm{Trp}}$ 氨酰化反应的 动力学参数}

图 1 中给出了水稻线粒体和人的两种野生型 $\mathrm{tRNA}^{\mathrm{Trp}}$ (图 1(a)和(i))的动力学常数的测试结果(相对 于野生型水稻线粒体 $\mathrm{tRNA} \mathrm{R}^{\mathrm{Trp}}$ 在 $k_{\mathrm{cat}} / K_{\mathrm{M}}$ 值上的变化). 枯草杆菌色氨酰 tRNA 合成酶氨酰化野生型水稻线粒 体 $\mathrm{tRNA}^{\mathrm{Tr}}$ 的反应中, $k_{\mathrm{cat}}$ 为 $1.33 \mathrm{~s}^{-1}, K_{\mathrm{M}}$ 为 $1.21 \mu \mathrm{mol} / \mathrm{L}$; 人色氨酰 $\mathrm{tRNA}$ 合成酶氨酰化水稻线粒体 $\mathrm{tRNA}^{\mathrm{Tr}}$
反应的 $k_{\mathrm{cat}}$ 为 $4.01 \times 10^{-4} \mathrm{~s}^{-1}, K_{\mathrm{M}}$ 为 $1.33 \mu \mathrm{mol} / \mathrm{L}$. 结果 表明, 人色氨酰 tRNA 合成酶催化水稻线粒体 $\mathrm{tRNA}^{\mathrm{Trp}}$ 氨酰化反应的能力微弱, 与野生型人 $\mathrm{tRNA}^{\mathrm{Trp}}$ 相比, $k_{\mathrm{cat}} / K_{\mathrm{M}}$ 值下降了 $99.97 \%$. 人 $\mathrm{tRNA}^{\mathrm{Trp}}$ 同 样也是枯草杆菌色氨酰 tRNA 合成酶的一个很差的底 物, 与野生型水稻线粒体 $\mathrm{tRNA}^{\mathrm{Trp}}$ 相比, 活力降低 了 $99.75 \%$.

\section{3 水稻线粒体 $\mathrm{TRNA}^{\mathrm{Trp}}$ 突变体的氨酰化反应动力 学参数}

图 1 中给出了水稻线粒体 7 种突变体的动力学常 数和相对于野生型水稻线粒体 $\mathrm{tRNA}^{\mathrm{Tr}}$ 的变化值(图 1(b) (h)). 结果表明, 与野生型水稻线粒体 $\mathrm{tRNA}^{\mathrm{Tr}}$ 相比, B. subtilis TrpRS 识别单点突变的突变体(MPH1, $\mathrm{MPH} 2$ 和 MPH3)的活力分别降低了 $88.67 \%, 73.67 \%$ 和 53.33\%(图 1(b) (d)); 识别双突变(MPH4, MPH5, MPH6)的能力降低更多, 分别降低了 $97.67 \%, 94.77 \%$ 和 $83.33 \%$ (图 1(e) (g)); 识别三突变体(MPH7)的活性 降低最多, 下降了 $99.79 \%$ (图 1(i)); 同样与野生型水 稻线粒体 $\mathrm{tRNA}^{\mathrm{Trp}}$ 相比, B. subtilis TrpRS 识别人野生 型 $\mathrm{tRNA}^{\mathrm{Trp}}$ 的活性下降 $99.75 \%$. 这说明以 B. subtilis $\mathrm{tRNA}^{\mathrm{Trp}}$ 为参照物, 水稻线粒体三突变体的种族特异 性已被完全翻转, 亦即水稻线粒体 $\mathrm{tRNA}^{\mathrm{Tr}}$ 的种族特 异性处于这 3 个位点上. 与野生型水稻线粒体 $\mathrm{tRNA}^{\mathrm{Trp}}$ 相比, 人 TrpRS 催化单点突变体 MPH1, $\mathrm{MPH} 2$ 和 $\mathrm{MPH} 3$ 的 $k_{\mathrm{cat}} / K_{\mathrm{M}}$ 的活力却分别提高了 6.7 , 5.1 和 4.0 倍; 识别双突变(MPH4, MPH5, MPH6)的 $k_{\text {cat }} / K_{\mathrm{M}}$ 值同样有很大的提高, 分别提高了 $10.0,10.0$ 和 6.7 倍; 识别三突变体(MPH7)的活性提高了 330 倍; 与野生型水稻线粒体 $\mathrm{tRNA}^{\mathrm{Tr}}$ 相比, 人 $\operatorname{TrpRS}$ 识别人 $\mathrm{tRNA}^{\mathrm{Trp}}$ 的活性提高了 3660 倍. 这说明以人 $\mathrm{tRNA}^{\mathrm{Trp}}$ 为参照物, 水稻线粒体 $\mathrm{tRNA}^{\mathrm{Trp}}$ 三突变体的种族特异 性也已被基本翻转, 亦即水稻线粒体 $\mathrm{tRNA}^{\mathrm{Tr}}$ 的种族 特异性, 主要处于这 3 个位点上. 即线粒体 $\mathrm{tRNA}^{\mathrm{Tr}}$ 其他位点的突变, 对其种族特异性的变化不会大于 12 倍 $(3660 / 330<12)$. 也即其他位点突变可能引起的 种族特异性改变, 将远小于上述 3 个位点突变所引起 的改变(330 倍).

综上所述, 可以得出, 与真核或原核生物细胞质 $\mathrm{tRNA}^{\mathrm{Trp}}$ 的情况相似 ${ }^{[8]}$, 水稻线粒体 $\mathrm{tRNA}^{\mathrm{Trp}}$ 的种族特 异性元件, 主要处于该 $\mathrm{tRNA}^{\mathrm{Tr}}$ 氨基酸接受茎上的 3 个位点, 即识别位碱基 G73, G1/U72 和 U5/A68(图 1). 


\section{3 讨论}

用体外转录的方法制备的 tRNA 分子内均不含 有存在于天然 tRNA 分子中的修饰碱基, 尽管这两种 不同来源的 tRNA 分子有所不同，但体外转录的 tRNA 分子仍可以作为它们相应的氨酰 tRNA 合成酶 的有效底物 ${ }^{[4,14]}$. 用枯草杆菌和人色氨酰 tRNA 合成 酶, 以体外转录的枯草杆菌和人的 $\mathrm{tRNA}^{\mathrm{Trp}}$ 作为底物, 测定它们的交叉氨酰化活力都有成功的报道 ${ }^{[5,8]}$. 由 于天然的水稻线粒体 $\mathrm{tRNA}^{\mathrm{Trp}}$ 仍未被纯化和检验氨 酰化活力, 所以我们采取了体外转录的方法制备水 稻线粒体 $\mathrm{tRNA}{ }^{\mathrm{Tr}}$, 并用于氨酰化活力检测.

在本研究中, $\mathrm{tRNA}^{\mathrm{Trp}}$ 的个性元件通过水稻线粒 体 $\mathrm{tRNA}^{\mathrm{Tr}}$ 的氨基酸接受茎向真核(人)方向的单点和 多点突变相结合的方法得到进一步的证实. 尽管在 枯草杆菌色氨酰 tRNA 合成酶氨酰化系统中, 反密 码子碱基被认为是枯草杆菌色氨酰 tRNA 合成酶识别 枯草杆菌 $\mathrm{tRNA}^{\mathrm{Tr}}$ 的一个重要个性元件 ${ }^{[15]}$, 但在本研 究中并未将它包括在内, 这是因为反密码子碱基对 所有 $\mathrm{tRNA}^{\mathrm{Trp}}$ 分子来说均是相同的, 因此也就不可能 对种属特异性氨酰化起作用.

Margulis 提出的连续内共生理论来解释线粒体 的起源 ${ }^{[16]}$, 在过去的 30 年里这一理论一直存在争议, 直到对核糖体 RNA 的排列顺序进行比较研究, 才首 次阐明了 proteobacteria 是线粒体的原始祖先 ${ }^{[17]}$. 随 后，系统发育提供的证据进一步支持了这一假设 $[18]$. 通过对真细菌的色氨酰 tRNA 合成酶或真核的色氨酰 tRNA 合成酶催化线粒体 $\mathrm{tRNA}^{\mathrm{Trp}}$ 的氨酰化活力的比 较研究, Chen等人 ${ }^{[19]}$ 认为, 线粒体 $\mathrm{tRNA}^{\mathrm{Trp}}$ 属于真细 菌的起源，为线粒体的起源提供了直接的实验依据. $\mathrm{Xu}$ 等人 ${ }^{[8]}$ 发现, 人 tRNA ${ }^{\operatorname{Trp}}$ 是枯草杆菌色氨酰 tRNA 合成酶的较差底物, 氨酰化反应的能力显著减弱. 类 似脯氨酰 tRNA 合成酶系统，人色氨酰 tRNA 合成酶 催化水稻线粒体 $\mathrm{tRNA}^{\mathrm{Tr}}$ 氨酰化反应也很弱 ${ }^{[19]}$. 何 新霞等人 20$]$ 研究了水稻线粒体 tRNATrp 的 3 个个性 元件 G73, U72 和 A68 向枯草杆菌 tRNATrp 的个性 元件 C73, G72 和 G68 的突变, 发现并不能明显提高 它被枯草杆菌色氨酰 tRNA 合成酶的催化活力. 本 研究发现, 当水稻线粒体 $\mathrm{tRNA}^{\mathrm{Trp}}$ 的 3 个个性元件 G73, U72 和 A68 具有了真核(人) $\mathrm{tRNA}^{\mathrm{Trp}}$ 的个性元 件 A73, C72 和 G68 后, 它被人色氨酰 tRNA 合成酶 的催化活力比野生型水稻线粒体提高了 330 倍, 而被 枯草杆菌色氨酰 tRNA 合成酶的催化活力下降了
99.79\%. 也就是说，这 3 个个性元件向真核方向改变 后, 赋予了该突变体部分真核 $\mathrm{tRNA}^{\mathrm{Trp}}$ 的特性. 从这 些实验数据我们推测, 三叶草结构中的氨基酸接受 茎对 $\mathrm{tRNA}^{\mathrm{Trp}}$ 的种属特异性氨酰化起重要作用. 本研 究通过单点突变和多点突变相结合的方法, 进一步 验证了这些个性元件. 这些实验数据进一步证实了 线粒体 $\mathrm{tRNA}^{\mathrm{Tr}}$ 属于真细菌起源的推论.

tRNA 是古老而具有多功能的分子, tRNA 及其类 似物中含有生命早期的痕迹, 因而系统地研究 tRNA 个性元件就有可能推测出遗传密码的起源及进化途 径 ${ }^{[10,12,20]}$. 另外, 利用 tRNA 与合成酶的相互作用区 域的识别特性，亦即氨基酸接受茎及识别位碱基，合 理地设计 tRNA 类似物(接受茎和识别位碱基)有可能 成为颇具潜力的治疗药物 ${ }^{[8,9]}$.

虽然突变体 MPH7 的氨基酸接受茎具有人 $\mathrm{tRNA}^{\operatorname{Trp}}$ 的个性元件, 赋予了部分真核 $\mathrm{tRNA}^{\mathrm{Trp}}$ 的特 性, 但是, 它的氨酰化活力仍只有野生型人 $\mathrm{tRNA}^{\mathrm{Tr}}$ 的 $10 \%(330 / 3660$, 图 1). 这个事实预示着在线粒体 $\mathrm{tRNA}^{\mathrm{Tr}}$ 中存在着除 G73, U72 和 A68 外的种属特异性 元件, 它限制了原核色氨酰 tRNA 合成酶对线粒体 $\mathrm{tRNA}^{\mathrm{Tr}}$ 的识别. 在枯草杆菌 $\mathrm{tRNA}^{\mathrm{Trp}}$ 的氨基酸接受茎 中已有类似的现象被发现 ${ }^{[9]}$. 有关水稻线粒体 $\mathrm{tRNA}^{\mathrm{Tr}}$ 中新的种属特异性元件研究有待进一步深人.

致谢本工作为浙江省自然科学基金资助项目 (批准号: 302103).

\section{参考文献}

1 Schimmel P. Aminoacyl-tRNA synthetases: General scheme of structure-functional relationships in the peptides and recognition of transfer RNAs. Annu Rev Biochem, 1987, 56: 125-158

2 Mc Clain W H. Rules that govern tRNA identity in protein synthesis. J Mol Biol, 1993, 234: 257-280[DOI]

3 Saks M E, Sampson J R, Abelson J N. The transfer RNA identity problem: A search for rules. Science, 1994, 263: 191-197

4 Hou Y M. Discriminating among the discriminator bases of tRNAs. Chem Biol, 1997, 4: 93-96[DOI]

5 Xue H, Shen W, Giegé R, et al. Identity elements of tRNA ${ }^{\mathrm{Trp}}$. J Biol Chem, 1993, 268: 9316-9322

6 Sprinzl M. Horn C, Brown M, et al. Compilation of tRNA sequences and sequences of tRNA genes. Nucleic Acids Res, 1998, 26: $148-153[\mathrm{DOI}]$

7 Shiba K, Schimmel P, Motegi H, et al. Human glycyl-tRNA synthetase wide divergence of primary structure from bacterial counterpart and species-specific aminoacylation. J Biol Chem, 1994, 269: $30049-30055$

$8 \mathrm{Xu} \mathrm{F}$, Chen X L, Xin L. Species: Specific differences in the opera- 
tional RNA code for aminoacylation of tRNA ${ }^{\text {Trp }}$. Nucleic Acids Res, 2001, 29: 4125-4133[DOI]

9 Xu F, Jiang G, Li W. Three G.C base pairs required for the efficient aminoacylation of tRNA ${ }^{\text {Trp }}$ by tryptophanyl-tRNA synthetase from Bacillus subtilis. Biochemistry, 2002, 41: 8087-8092[DOI]

$10 \mathrm{Xu} \mathrm{F}$, Jia J, Jin Y X, et al. High-level expression and single-step purification of human tryptophanyl-tRNA synthetase. Protein Exp Purif, 2001, 23: 296-300[DOI]

11 Sampson J R, Uhlenbeck O C. Biochemical and physical characterization of an unmodified yeast phenylalanine transfer RNA transcribed in vitro. Proc Natl Acad Sci USA, 1988, 85: 10331038

12 Wen S, Chow K C, Wong J T. High-level expression of Bacillus Subtilis tryptophanyl-tRNA synthetase in Escherichia coli. Biochem Cell Biol, 1990, 68: 492-495

13 Xu Z, Love M L, Ma L Y. Tryptophanyl-tRNA synthetase from Bacillus.subtilis. J Biol Chem, 1989, 264: 4304-4311

14 Crothers D M, Seno T, Söll D. Is there a discriminator site in transfer RNA? Proc Natl Acad Sci USA, 1972, 69: 3063-3067
15 Eriani G, Delarue M, Poch O, et al. Partition of tRNA synthetases into two classes based on mutually exclusive sets of sequence motifs. Nature, 347, 1990: 203-206[DOI]

16 Margulis L. Symbiosis in Cell Evolution. San Francisco: Freeman WH Company, 1981. 1-11

17 Hipps D, Shiba K, Henderson B, et al. Operational RNA code for amino acids: species-specific aminoacylation of minihelices switched by a single nucleotide. Proc Natl Acad Sci USA, 1995, 92: $5550-5552$

18 Yang D, Oyaizu Y, Oyaizu H, et al. Mitochondrial orgins. Proc Natl Acad Sci USA, 1985, 82: 4443-4447

19 Chen L, Jin Y X, Wang D B. Mitochondrial tRNA ${ }^{\text {Trp }}$ imply a eubacterial origin. Acta Biochem Biophys Sin, 2000, 32: 100 104

20 He X X, Xu F, Chen L. Cloning and characterization of three mutants from Oryza sativa mitochondrial tRNA ${ }^{\text {Trp }}$. Chin J Biochem Mol Biol, 2001, 17: 453-457

(2005-11-21 收稿, 2006-01-23 接受) 